\title{
Temperatura do Ar no Desenvolvimento Reprodutivo de Cultivares de Oliveira
}

\author{
Fabrina Bolzan Martins ${ }^{1}$ (D), Mábele de Cássia Ferreira ${ }^{1}$, Luiz Fernando de Oliveira da Silva ${ }^{2}$, \\ Vinícius Ribeiro Moura Ramalho ${ }^{1}$, Emerson Dias Gonçalves ${ }^{2}$ \\ ${ }^{1}$ Instituto de Recursos Naturais, Universidade Federal de Itajubá, Itajubá, MG, Brasil. \\ ${ }^{2}$ Empresa de Pesquisa Agropecuária de Minas Gerais, Campo Experimental de Maria da Fé, \\ Maria da Fé, MG, Brasil.
}

Recebido em: 1 de Abril de 2018 - Aceito em: 20 de Novembro de 2018

\begin{abstract}
Resumo
A temperatura do ar é uma das variáveis que exerce maior influencia o desenvolvimento reprodutivo da oliveira. O conhecimento dos efeitos da temperatura é vital para determinar o manejo das plantas no campo e escolher as cultivares mais adaptadas aos locais de cultivo. Neste estudo, a necessidade térmica ( $S T a,{ }^{\circ} \mathrm{C}$.dia) foi utilizada para identificar a influência da temperatura em três subperíodos de desenvolvimento reprodutivo (floração, frutificação e maturação) em dez cultivares de oliveira, e estimar a temperatura basal inferior $\left(\mathrm{Tb},{ }^{\circ} \mathrm{C}\right)$ necessária no cômputo da $S T a$. Os dados utilizados referem-se às variáveis início, fim e de duração (em dias) para os anos agrícolas de 2008/2009, 2009/2010 e 2010/

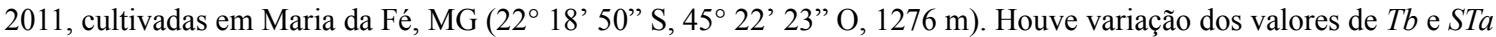
entre as cultivares e subperíodos de desenvolvimento reprodutivo. As $T b$ 's variaram para a floração: $7,8^{\circ} \mathrm{C}$ a $16,1{ }^{\circ} \mathrm{C}$, frutificação: $13,3{ }^{\circ} \mathrm{C}$ a $14,3{ }^{\circ} \mathrm{C}$, e maturação: $7,1{ }^{\circ} \mathrm{C}$ a $17,2{ }^{\circ} \mathrm{C}$. Já a frutificação é o subperíodo que demanda maior necessidade térmica, independentemente da cultivar de oliveira.
\end{abstract}

Palavras-chave: Olea europaea L., temperatura basal inferior, fenologia, Minas Gerais.

\section{Air Temperature on Reproductive Development of Olive Cultivars}

\begin{abstract}
The air temperature is the meteorological variable that most influences the reproductive development of the olive tree. The knowledge of the effects of the air temperature is important to optimize the management strategies and to choose the cultivars are more adapted to climatic condition. The thermal time $\left(S T a,{ }^{\circ} \mathrm{C}\right.$.day) was used to identify the influence of temperature on three subperiods of reproductive development (flowering, fruit ripening and maturation) in ten olive cultivars, and to estimate the base temperature $\left(\mathrm{Tb},{ }^{\circ} \mathrm{C}\right)$ to $S T a$. The data used refer to the variables beginning, end and duration (in days) for the agricultural years 2008/2009,2009/2010 and 2010/2011, cultivated in Maria da Fé, MG (22 $18^{\prime} 50^{\prime \prime} \mathrm{S}, 45^{\circ} 22^{\prime} 23^{\prime}$ ' W, $1276 \mathrm{~m}$ ). There was variation of $T b$ and $S T a$ values between cultivars and subperiods of reproductive development. $\mathrm{Tb}$ 's varied for flowering: $7.8^{\circ} \mathrm{C}$ to $16.1{ }^{\circ} \mathrm{C}$, fruit ripening: $13.3{ }^{\circ} \mathrm{C}$ to $14.3{ }^{\circ} \mathrm{C}$, and maturation: $7.1{ }^{\circ} \mathrm{C}$ to $17.2{ }^{\circ} \mathrm{C}$. The fruit ripening is the subperiod that needs greater thermal time, independently of olive cultivars.
\end{abstract}

Keywords: Olea europaea L., base temperature, phenology, Minas Gerais.

\section{Introdução}

A oliveira (Olea europaea L., família Oleaceae) é uma árvore frutífera de clima temperado, originária do norte da Síria e sul da Turquia e vem sendo plantada em grande escala em diversas regiões do mundo, inclusive em locais fora do seu centro de domesticação (Bacia do Mediterrâneo), como no Brasil (Martins et al. 2014; Wrege et al. 2015). Isso ocorre devido a grande demanda mundial (Garcia et al. 2018) e pelos usos múltiplos da oliveira,

Autor de correspondência: Fabrina Bolzan Martins, fabrinabm@gmail.com. 
como a finalidade paisagística e de recuperação de áreas degradadas (Vieira Neto et al. 2008).

Em 2017, a importação de azeite no Brasil atingiu aproximadamente 55.077 toneladas, colocando o país como sétimo maior importador mundial (TRADEMAP, 2018). Atualmente, a oliveira é plantada principalmente nas regiões sul e sudeste do Brasil (Martins et al. 2014), porém os cultivos ainda são incipientes sem escala comercial, como um complemento na renda dos agricultores, já que as condições climáticas dessas regiões diferem das condições do seu local de origem.

Nesse sentido, as principais condições limitantes à olivicultura são a ocorrência de eventos extremos associados principalmente à temperatura do ar (Garcia-Mozo et al. 2010; Moriondo et al. 2015; Pérez-López et al. 2008; Streck et al. 2007). Essa limitação é agravada em função da época de ocorrência dos eventos extremos (Garcia et al. 2018), principalmente quando ocorrem nos subperíodos de florescimento, polinização, fixação e maturação dos frutos (Aguilera et al. 2015; Wrege et al. 2015) e do estádio de desenvolvimento. Além disso, o desconhecimento das necessidades térmicas da oliveira em condições climáticas fora dos locais de origem são entraves à viabilização da expansão da olivicultura no Brasil (Martins et al. 2012; Martins et al. 2014; Wrege et al. 2015).

A maneira mais simples e frequentemente usada para descrever o efeito da temperatura do ar sobre o desenvolvimento da oliveira é pela soma térmica, cuja unidade é ${ }^{\circ}$ C.dia (Pérez-López et al. 2008; Souza e Martins, 2014). A soma térmica é baseada no acúmulo térmico diário dentro dos quais a planta se desenvolve, sendo delimitada pelas temperaturas basais superior $(T B)$ e inferior (Tb) (Freitas et al. 2017). A $T B$ e $T b$ representam, respectivamente, a temperatura acima e abaixo da qual o desenvolvimento é nulo ou desprezível para fins de cálculo (Souza e Martins, 2014). Geralmente, a TB assume valores elevados, os quais dificilmente são atingidos durante o ciclo de desenvolvimento no campo (Ferreira et al. 2019) e por essa razão a $T B$ não é computada em alguns métodos de soma térmica. Já a $T b$ assume valores baixos, os quais ocorrem principalmente durante o inverno, sendo bastante utilizada na clássica equação de cálculo da soma térmica (Freitas et al. 2017). Adicionalmente, a Tb é importante na quantificação coerente do número de horas frio que é uma exigência da oliveira para interromper a dormência fisiológica e iniciar a diferenciação dos botões florais (Wrege et al. 2015). Assim, para uma estimativa precisa do desenvolvimento e do conhecimento das necessidades térmicas de determinada espécie, é necessário determinar a $T b$ adequada e escolher o método de soma térmica mais pertinente (Ferreira et al. 2019; Freitas et al. 2017; PérezLópez et al. 2008).

$\mathrm{Na}$ maioria dos casos, a $\mathrm{Tb}$ é determinada por métodos estatísticos com base em dados de observações fenológicas e de temperatura média do ar (Ferreira et al. 2019;
Freitas et al. 2017). Existe uma variedade de métodos estatísticos utilizados para estimar a $T b$, sendo os mais utilizados: desvio padrão em dias e graus-dia, coeficiente de variação em dias e graus-dia, o coeficiente de regressão, o $\mathrm{X}$ intercepto e o menor quadrado médio do erro (Ferreira et al., 2019; Freitas et al. 2017; Lago et al. 2009; Souza e Martins, 2014; Yang et al. 1995). Ressaltando que os valores de $T b$ variam em função da espécie, cultivares e também para as fases de desenvolvimento no qual a planta se encontra (Lago et al. 2009).

Estudos sobre a estimativa da $T b$ e sobre a necessidade térmica, baseado na soma térmica, são amplamente utilizados para culturas agrícolas, ornamentais, medicinais e culturas perenes como a oliveira (Martins et al. 2012; Martins et al. 2014; Pérez-López et al. 2008; Souza e Martins, 2014). No entanto, não existem estudos dessa natureza para a oliveira considerando as fases do desenvolvimento reprodutivo que constituiu o esforço para a realização deste trabalho.

Dessa forma, o objetivo deste trabalho foi estimar a $T b$ e determinar as necessidades térmicas no período reprodutivo (florescimento, frutificação e maturação) em 10 cultivares de oliveira para Maria da Fé (MG).

\section{Material e Métodos}

Os dados utilizados referem-se a fase de desenvolvimento reprodutivo composto pelos subperíodos: floração, frutificação e maturação. Para quantificar esses dados foram utilizadas as variáveis data de início, fim e de duração (em dias), baseado no calendário civil, de cada subperíodo disponíveis em Oliveira et al. (2012) (Tabela 1). Utilizou essa base de dados fenológicos, pois é a única base existente relacionados à fenologia do desenvolvimento reprodutivo de diferentes cultivares de oliveira em Minas Gerais. Esses dados são referentes aos anos agrícolas de 2008/2009, 2009/2010 e 2010/2011 de 10 cultivares de oliveira (consideradas de ciclo precoce, médio e tardio) cultivadas em Maria da Fé, MG (22 $18^{\prime} 50^{\prime \prime} \mathrm{S}, 45^{\circ} 22^{\prime} 23^{\prime}$ ' $\mathrm{O}, 1276 \mathrm{~m}$ de altitude) (Tabela 1). Os autores consideraram o início da floração a partir da emissão do botão floral e o final com $95 \%$ das flores abertas. Para a frutificação, seu início foi considerado com $5 \%$ dos frutos pegos, conforme Sanz-Cortez et al. (2002).

As plantas utilizadas neste levantamento fenológico fazem parte do Banco de Germoplasma da Empresa de Pesquisa Agropecuária de Minas Gerais - EPAMIG, em Maria da Fé, o qual foi instalado no ano de 2005 no espaçamento de $7 \mathrm{~m}$ entre linhas e $5 \mathrm{~m}$ entre plantas, sendo 6 plantas de cada cultivar: Alto D'Ouro, Arbequina, Galega, Grappolo 541, Grappolo 561, Grappolo 575, Negroa, Penafiel, Ropades 392 e Santa Catalina. As plantas apresentavam tamanho de 5 metros de altura, conduzidas de forma livre e manejadas conforme orientações de Silva et al. (2012), não sendo utilizado irrigação já que o local 
Tabela 1 - Caracterização da temperatura do ar e da precipitação acumulada (P) durante os três anos agrícolas (2008/2009, 2009/2010, 2010/2011) do experimento a campo e duração de cada fase de desenvolvimento reprodutivo, correspondente ao período que se estende desde o dia de início até o término dos subperíodos floração, frutificação e maturação para as 10 cultivares de oliveira. Maria da Fé, MG, $2008-2011$.

\begin{tabular}{|c|c|c|c|c|c|c|}
\hline \multirow[t]{2}{*}{ Cultivar } & \multirow[t]{2}{*}{ Subperíodo } & \multicolumn{3}{|c|}{ Média da temperatura do $\operatorname{ar}\left({ }^{\circ} \mathrm{C}\right)$} & \multirow[t]{2}{*}{$\mathrm{P}(\mathrm{mm})$} & \multirow[t]{2}{*}{ Duração média da fase de desenvolvimento* } \\
\hline & & Mínima & Média & Máxima & & \\
\hline \multicolumn{7}{|c|}{ Ano agrícola 2008/2009 } \\
\hline \multirow[t]{3}{*}{ Alto D’Ouro } & Floração & 8,9 & 16,7 & 24,5 & 114 & 60 \\
\hline & Frutificação & 13,3 & 19,3 & 25,4 & 1223 & 148 \\
\hline & Maturação & 16 & 22,2 & 28,3 & 204 & 17 \\
\hline \multirow[t]{3}{*}{ Arbequina } & Floração & 8,6 & 16,5 & 24,5 & 180 & 56 \\
\hline & Frutificação & 12,7 & 19 & 25,3 & 1158 & 153 \\
\hline & Maturação & 15,6 & 21,9 & 28,1 & 295 & 26 \\
\hline \multirow[t]{3}{*}{ Galega } & Floração & 10,3 & 17,5 & 24,8 & 148 & 49 \\
\hline & Frutificação & 14,1 & 20 & 25,9 & 1383 & 146 \\
\hline & Maturação & 16,4 & 23 & 29,5 & 127 & 17 \\
\hline \multirow[t]{3}{*}{ Grappolo 541} & Floração & 8,5 & 16,6 & 24,8 & 107 & 54 \\
\hline & Frutificação & 13,3 & 19,3 & 25,4 & 1223 & 148 \\
\hline & Maturação & 15,9 & 22,2 & 28,6 & 259 & 23 \\
\hline \multirow[t]{3}{*}{ Grappolo 561} & Floração & 10 & 17,5 & 25,1 & 175 & 80 \\
\hline & Frutificação & 13,7 & 19,7 & 25,6 & 1303 & 146 \\
\hline & Maturação & 15,7 & 22,9 & 30,2 & 118 & 11 \\
\hline \multirow[t]{3}{*}{ Grappolo 575} & Floração & 8,8 & 16,7 & 24,5 & 160 & 53 \\
\hline & Frutificação & 13,2 & 19,4 & 25,6 & 1354 & 168 \\
\hline & Maturação & 15,7 & 22,9 & 30,2 & 172 & 18 \\
\hline \multirow[t]{3}{*}{ Negroa } & Floração & 8,9 & 16,7 & 24,5 & 114 & 60 \\
\hline & Frutificação & 13,3 & 19,3 & 25,4 & 1223 & 148 \\
\hline & Maturação & 16 & 22,2 & 28,5 & 257 & 21 \\
\hline \multirow[t]{3}{*}{ Penafiel } & Floração & 9,2 & 16,9 & 24,7 & 92 & 50 \\
\hline & Frutificação & 14,2 & 19,8 & 25,3 & 1137 & 142 \\
\hline & Maturação & 22,1 & 25,1 & 28,2 & 263 & 24 \\
\hline \multirow[t]{3}{*}{ Ropades 392} & Floração & 8,7 & 16,7 & 24,7 & 126 & 56 \\
\hline & Frutificação & 13,2 & 19,3 & 25,5 & 1223 & 153 \\
\hline & Maturação & 16 & 22,2 & 28,5 & 257 & 21 \\
\hline \multirow[t]{3}{*}{ Santa Catalina } & Floração & 10,1 & 17,6 & 25,2 & 171 & 70 \\
\hline & Frutificação & 13,6 & 19,6 & 25,6 & 1348 & 153 \\
\hline & Maturação & 16 & 22,7 & 29,4 & 188 & 23 \\
\hline \multicolumn{7}{|c|}{ Ano agrícola 2009/2010 } \\
\hline \multirow[t]{3}{*}{ Alto D’Ouro } & Floração & 10,5 & 17,5 & 24,6 & 264 & 66 \\
\hline & Frutificação & 13,9 & 19,6 & 25,4 & 1177 & 138 \\
\hline & Maturação & 16,1 & 23,4 & 30,7 & 40 & 15 \\
\hline \multirow[t]{3}{*}{ Arbequina } & Floração & 10,1 & 17,3 & 24,5 & 228 & 56 \\
\hline & Frutificação & 13,3 & 19,2 & 25,1 & 1176 & 148 \\
\hline & Maturação & 16 & 22,8 & 29,5 & 113 & 31 \\
\hline \multirow[t]{3}{*}{ Galega } & Floração & 10 & 17,0 & 24,1 & 174 & 57 \\
\hline & Frutificação & 13,6 & 19,5 & 25,4 & 1251 & 155 \\
\hline & Maturação & 16,1 & 22,5 & 28,8 & 140 & 25 \\
\hline \multirow[t]{3}{*}{ Grappolo 541} & Floração & 10,1 & 17,2 & 24,2 & 249 & 82 \\
\hline & Frutificação & 13,9 & 19,7 & 25,5 & 1179 & 143 \\
\hline & Maturação & 16,6 & 23,0 & 29,3 & 63 & 18 \\
\hline \multirow[t]{2}{*}{ Grappolo 561} & Floração & 10,3 & 17,3 & 24,3 & 276 & 85 \\
\hline & Frutificação & 13,3 & 19,2 & 25,2 & 1249 & 162 \\
\hline
\end{tabular}


Tabela 1 - continua

\begin{tabular}{|c|c|c|c|c|c|c|}
\hline \multirow[t]{2}{*}{ Cultivar } & \multirow[t]{2}{*}{ Subperíodo } & \multicolumn{3}{|c|}{ Média da temperatura do ar $\left({ }^{\circ} \mathrm{C}\right)$} & \multirow[t]{2}{*}{$\mathrm{P}(\mathrm{mm})$} & \multirow[t]{2}{*}{ Duração média da fase de desenvolvimento* } \\
\hline & & Mínima & Média & Máxima & & \\
\hline & Maturação & 16 & 23,2 & 30,4 & 40 & 20 \\
\hline \multirow[t]{3}{*}{ Grappolo 575} & Floração & 10,3 & 17,3 & 24,3 & 276 & 85 \\
\hline & Frutificação & 13,4 & 19,5 & 25,5 & 1289 & 179 \\
\hline & Maturação & 16,9 & 21,7 & 26,4 & 120 & 13 \\
\hline \multirow[t]{3}{*}{ Negroa } & Floração & 10,5 & 17,5 & 24,5 & 264 & 61 \\
\hline & Frutificação & 13,9 & 19,8 & 25,6 & 1131 & 140 \\
\hline & Maturação & 16,7 & 23,3 & 29,8 & 50 & 15 \\
\hline \multirow[t]{3}{*}{ Penafiel } & Floração & 11 & 17,6 & 24,1 & 245 & 72 \\
\hline & Frutificação & 13,1 & 19,4 & 25,6 & 1263 & 168 \\
\hline & Maturação & 18,2 & 24,1 & 29,9 & 50 & 13 \\
\hline \multirow[t]{3}{*}{ Ropades 392} & Floração & 10,3 & 17,3 & 24,3 & 231 & 66 \\
\hline & Frutificação & 13,7 & 19,6 & 25,4 & 1248 & 157 \\
\hline & Maturação & 16,6 & 23,5 & 30,4 & 38 & 16 \\
\hline \multirow[t]{3}{*}{ Santa Catalina } & Floração & 10,6 & 17,6 & 24,7 & 270 & 69 \\
\hline & Frutificação & 13,8 & 19,7 & 25,7 & 1223 & 163 \\
\hline & Maturação & 16 & 21,2 & 26,5 & 215 & 16 \\
\hline \multicolumn{7}{|c|}{ Ano agrícola 2010/2011 } \\
\hline \multirow[t]{3}{*}{ Alto D’Ouro } & Floração & 9,4 & 17,3 & 25,3 & 165 & 51 \\
\hline & Frutificação & 14 & 20,2 & 26,3 & 1163 & 133 \\
\hline & Maturação & 11,6 & 17,3 & 23 & 70 & 11 \\
\hline \multirow[t]{3}{*}{ Arbequina } & Floração & 7,5 & 16,1 & 24,7 & 97 & 65 \\
\hline & Frutificação & 12,7 & 19,2 & 25,7 & 1195 & 155 \\
\hline & Maturação & 13,8 & 20,1 & 26,4 & 132 & 22 \\
\hline \multirow[t]{3}{*}{ Galega } & Floração & 9,4 & 17,3 & 25,3 & 165 & 51 \\
\hline & Frutificação & 13,9 & 20,0 & 26,2 & 1257 & 146 \\
\hline & Maturação & 13,7 & 19,2 & 24,6 & 288 & 21 \\
\hline \multirow[t]{3}{*}{ Grappolo 541} & Floração & 8 & 16,3 & 24,6 & 165 & 84 \\
\hline & Frutificação & 13,6 & 19,6 & 25,7 & 1303 & 152 \\
\hline & Maturação & 16,1 & 21,9 & 27,7 & 274 & 19 \\
\hline \multirow[t]{3}{*}{ Grappolo 561} & Floração & 10,7 & 17,6 & 24,6 & 203 & 45 \\
\hline & Frutificação & 14 & 20,2 & 26,3 & 1163 & 133 \\
\hline & Maturação & 12,1 & 18,0 & 23,9 & 85 & 13 \\
\hline \multirow[t]{3}{*}{ Grappolo 575} & Floração & 10,7 & 17,6 & 24,5 & 165 & 35 \\
\hline & Frutificação & 12,7 & 19,1 & 25,5 & 1306 & 173 \\
\hline & Maturação & 16,1 & 22,8 & 29,4 & 208 & 15 \\
\hline \multirow[t]{3}{*}{ Negroa } & Floração & 9,4 & 17,3 & 25,3 & 165 & 51 \\
\hline & Frutificação & 13,9 & 20,0 & 26 & 1257 & 140 \\
\hline & Maturação & 12,8 & 18,9 & 25 & 85 & 16 \\
\hline \multirow[t]{3}{*}{ Penafiel } & Floração & 12,3 & 18,7 & 25 & 130 & 36 \\
\hline & Frutificação & 12,9 & 19,3 & 25,7 & 1342 & 156 \\
\hline & Maturação & 29,6 & 30,5 & 31,5 & 173 & 6 \\
\hline \multirow[t]{3}{*}{ Ropades 392} & Floração & 10,5 & 17,8 & 25,1 & 165 & 39 \\
\hline & Frutificação & 13,9 & 20,0 & 26,1 & 1257 & 143 \\
\hline & Maturação & 12,8 & 18,7 & 24,6 & 114 & 16 \\
\hline \multirow[t]{3}{*}{ Santa Catalina } & Floração & 10,6 & 17,5 & 24,4 & 165 & 40 \\
\hline & Frutificação & 13,4 & 19,6 & 25,8 & 1317 & 160 \\
\hline & Maturação & 16,4 & 23,1 & 29,8 & 205 & 12 \\
\hline
\end{tabular}

*Valores extraídos de Oliveira et al. (2012). 
apresenta precipitação suficiente para o desenvolvimento das plantas (média climatológica da precipitação acumulada $\approx 1600 \mathrm{~mm}$ ) (Silva e Reboita, 2014; Garcia et al. 2018).

As variáveis meteorológicas, temperatura mínima $(T m)$ e máxima $(T M)$ diárias do ar, foram obtidas de uma estação meteorológica convencional pertencente ao Instituto Mineiro de Gestão das Águas - IGAM, localizada na EPAMIG a $150 \mathrm{~m}$ da coleta dos dados de Oliveira et al. (2012).

Para a estimativa da $T b$ foram utilizadas as metodologias tradicionais e modificadas propostas por Yang et al. (1995), utilizadas por Ferreira et al. (2019); Lago et al. (2009) e Souza e Martins (2014).

Primeiramente calculou-se a soma térmica diária $\left(S T d_{i},{ }^{\circ} \mathrm{C} . \mathrm{dia}\right)$ por:

$$
S T d_{i}=\left(\frac{T M+T m}{2}\right)-T b
$$

em que $T M$ é a temperatura máxima diária do $\operatorname{ar}\left({ }^{\circ} \mathrm{C}\right), T m$ é a temperatura mínima diária do ar $\left({ }^{\circ} \mathrm{C}\right)$ e $T b$ é a temperatura basal e inferior de cada cultivar de oliveira. Para o cálculo da $S T d_{i}$ foi utilizada uma série de $T b$ 's variando de $0{ }^{\circ} \mathrm{C}$ a $30^{\circ} \mathrm{C}$, em intervalos de $0,5^{\circ} \mathrm{C}$ (Freitas et al. 2017).

A soma térmica acumulada, denominada necessidade térmica (STa, ${ }^{\circ} \mathrm{C}$.dia) a partir da data de início de cada subperíodo e de cada ano agrícola, foi obtida pelo somatório da $S T d_{i}$ :

$$
S T a=\sum S T d_{i}
$$

As seis metodologias tradicionais para a estimativa da Tb foram (Yang et al. 1995; Souza e Martins, 2014): desvio padrão em graus-dia $\left(D P_{g d}\right)$, desvio padrão em dias $\left(D P_{d}\right)$, coeficiente de variação em dias $\left(C V_{d}\right)$, coeficiente de variação em graus-dia $\left(C V_{g d}\right)$, coeficiente de regressão $(C R)$, e desenvolvimento relativo $(D R)$. Todos os métodos foram aplicados separadamente para cada cultivar e subperíodo de desenvolvimento reprodutivo.

$\mathrm{O} D P_{g d}$ considera a $T b$ como a resultante no menor desvio padrão em soma térmica, ou seja, a variação resultante em soma térmica, usando-se uma série de anos agrícolas, deve ser mínima (Fagundes et al. 2010; Lago et al. 2009):

$$
D P g d=\sqrt{\frac{\sum_{i=1}^{n}\left(S T a_{i}-M S T a\right)^{2}}{n-1}}
$$

em que $S T a_{i}$ é a soma térmica acumulada no $i$-ésimo ano agrícola utilizando uma série de $T b ; M S T a$ é a média da soma térmica acumulada para todos $i$-ésimos anos agrícolas; $n$ é o número anos agrícolas.
O $D P_{d}$ considera a $T b$ como a resultante no menor desvio padrão entre os diferentes anos agrícolas (Ferreira et al. 2019; Souza e Martins, 2014):

$$
D P_{d}=\frac{D P_{g d}}{\bar{x}-T b}
$$

em que $\bar{x}$ é a temperatura média do ar dos $i$ anos agrícolas; $T b$ é a temperatura basal inferior $\left({ }^{\circ} \mathrm{C}\right)$.

No $C V_{d}$ a $T b$ é aquela que decorre em um menor coeficiente de variação em dias (\%), obtido pela relação entre o desvio padrão em dias e o número de dias de cada subperíodo (Souza e Martins, 2014; Yang et al. 1995):

$$
C V_{d}=\frac{D P_{d}}{\bar{x} d} \cdot 100
$$

em que $\bar{x} d$ é a média do número de dias para atingir determinado subperíodo reprodutivo entre os anos agrícolas.

$\mathrm{O} C V_{g d}$ considera a $T b$ aquela que apresenta o menor coeficiente de variação com relação a soma térmica necessária para atingir cada subperíodo reprodutivo (Souza e Martins, 2014):

$$
C V_{g d}=\frac{D P_{g d}}{M S T a} \cdot 100
$$

O $C R$ baseia-se na relação linear existente entre a temperatura média do ar $\left(\right.$ Tmed,$\left.{ }^{\circ} \mathrm{C}\right)$ durante o subperíodo reprodutivo e as $S T a_{i}$ necessárias para atingir o subperíodo reprodutivo:

$$
S T a_{i}=a \cdot T m e d+b
$$

em que $a$ é o coeficiente angular da regressão linear, $b$ é o coeficiente linear.

O coeficiente angular $(a)$ da regressão linear simples indica o valor da $T b$ correta. Valor de ' $a$ ' positivo indica que a $T b$ usada para calcular $S T a$ é elevado e maior que o valor verdadeiro, ' $a$ ' negativo indica valor de $T b$ baixo e menor que o valor verdadeiro, ' $a$ ' igual zero (ou próximo) indica o valor de $T b$ correto (Fagundes et. al., 2010; Souza e Martins, 2014).

$\mathrm{O} D \mathrm{R}$ considera a $T b$ como aquela em que não ocorre desenvolvimento, sendo estimada pelo prolongamento da regressão linear simples entre o $D R$ em função da Tmed $(T b=-b / a)$ (Fagundes et al. 2010):

$$
D R=a \cdot \text { Tmed }+b, \text { sendo } \quad D R=100 / N
$$

em que 100 é um valor arbitrário de ponderação; $N$ é o número de dias de duração de cada subperíodo de desenvolvimento reprodutivo em cada ano agrícola.

As três metodologias modificadas para a estimativa da $T b$ foram (Ferreira et al. 2019; Yang et al. 1995): 
Menor desvio padrão em graus-dia $\left(D P_{g d}\right)$ :

$$
T b=\frac{\sum_{i=1}^{n}(\text { Tmed } \cdot N) \cdot \sum_{i=1}^{n} N-n \cdot \sum_{i=1}^{n}\left(N^{2} \cdot \text { Tmed }\right)}{\left(\sum_{i=1}^{n} N\right)^{2}-n \cdot \sum_{i=1}^{n} N^{2}}
$$

Coeficiente de variação em graus-dia $\left(C V_{g d}\right)$ :

$$
T b=\frac{\sum_{i=1}^{n}\left(\text { Tmed } \cdot N^{2}\right) \cdot \sum_{i=1}^{n}(\text { Tmed } \cdot N)-\sum_{i=1}^{n} N \cdot \sum_{i=1}^{n}\left(N^{2} \cdot \text { Tmed }^{2}\right)}{\sum_{i=1}^{n} N^{2} \sum_{i=1}^{n}(\text { Tmed } \cdot N)-\sum_{i=1}^{n} N \cdot \sum_{i=1}^{n}\left(N^{2} \cdot \text { Tmed }\right)}
$$

Coeficiente de regressão $(C R)$ :

$$
T b=\frac{\sum_{i=1}^{n}(\text { Tmed }) \sum_{i=1}^{n}(\text { Tmed } \cdot N)-n \cdot \sum_{i=1}^{n}\left(N \cdot \text { Tmed }^{2}\right)}{\sum_{i=1}^{n} N \cdot \sum_{i=1}^{n}(\text { Tmed })-n \cdot \sum_{i=1}^{n}(N \cdot \text { Tmed })}
$$

em que $T$ é a temperatura média do ar de todos os i-ésimos anos agrícolas $\left({ }^{\circ} \mathrm{C}\right) ; N$ é a duração em dias dos i-ésimos anos agrícolas em cada subperíodo; Tmed é a temperatura média do ar durante os i-ésimos anos agrícolas em cada subperíodo $\left({ }^{\circ} \mathrm{C}\right) ; n$ é o número de anos agrícolas.

Devido às diferenças entre os valores de $T b$ encontrados entre as nove metodologias (Eqs. (3)-(11)), o valor final da $T b$ para cada cultivar e subperíodo reprodutivo foi obtido pela média aritmética dos valores de $T b$, conforme recomendações de Ferreira et al. (2019), Lago et al. (2009) e Souza e Martins (2014).

Com relação à necessidade térmica $\left(S T a,{ }^{\circ} \mathrm{C}\right.$.dia $)$, os dois métodos utilizados neste estudo foram os que consideram apenas a $T b$, devido a impossibilidade de estimar as demais temperaturas cardinais (ótima e basal superior) com os dados fenológicos disponíveis em Oliveira et al. (2012). Os métodos utilizados foram (Freitas e Martins, 2019; Rosa et al. 2009):

Método 1 (M1): Quando Tmed < Tb, considera Tmed $=T b$ e quando Tmed $\geq T b$,calcula:

$$
S T d_{i}=\text { Tmed }-T b \quad \cdot 1 \mathrm{dia}
$$

Método 2 (M2): Quando $T m \leq T b$, considera $T m=T b$ e quando $T m e d \geq T b$, calcula:

$$
S T d_{i}=T m e d-T b \quad \cdot 1 \mathrm{dia}
$$

em que Tmed é a temperatura média diária do ar $\left({ }^{\circ} \mathrm{C}\right)$, obtida pela média aritmética das temperaturas máximas
$(T M)$ e mínimas do ar $(T m)$ e $T b$ é a temperatura basal inferior $\left({ }^{\circ} \mathrm{C}\right)$ de cada cultivar e subperíodo reprodutivo.

Para a escolha do melhor método de STa, utilizou-se o coeficiente de variação (CV, \%), conforme recomendações de Freitas e Martins (2019), Rosa et al. (2009) e Streck et al. (2007). Quanto menor o valor de CV, mais adequado é o método de $S T a$.

Posteriormente, o melhor método de STa foi submetido a análise de variância (ANOVA), e comparados pelo teste de Scott-Knott a 5\% de probabilidade, para verificar o efeito das fontes de variação - cultivares e subperíodos de desenvolvimento reprodutivo (floração, frutificação e maturação). Os testes foram realizados no software SISVAR 5.3 (Ferreira, 2011).

\section{Resultados e Discussão}

O cultivo nos diferentes anos agrícolas proporcionou uma análise pormenorizada do período reprodutivo das cultivares de oliveira em relação às distintas condições meteorológicas para a região estudada (Tabela 1), as quais foram importantes na estimativa da $T b$, sendo premissa neste tipo de estudo (Ferreira et al. 2019; Freitas et al. 2017; Rosa et al. 2009). As Tm e TM absolutas do ar foram de, respectivamente, $2,7^{\circ} \mathrm{C}$ e $31,8^{\circ} \mathrm{C}$, para o ano agrícola $2008 / 2009 ; 1,3{ }^{\circ} \mathrm{C}$ e $32,2^{\circ} \mathrm{C}$ para $2009 / 2010$ e $0,7^{\circ} \mathrm{C}$ e $31,9^{\circ} \mathrm{C}$ para $2010 / 2011$. Já as médias de $T m$ e $T M$ foram de $12,6^{\circ} \mathrm{C}$ e $25,5^{\circ} \mathrm{C}(2008 / 2009), 13,4^{\circ} \mathrm{C}$ e $26,0^{\circ} \mathrm{C}$ (2009/2010) e $12,1^{\circ} \mathrm{C}$ e $26,1^{\circ} \mathrm{C}(2010 / 2011)$. Com relação à precipitação acumulada $(P)$, observou pouca variação entre os três anos agrícolas, sendo os maiores valores observados durante a frutificação (média de $1244 \mathrm{~mm}$ entre as cultivares), que ocorre desde a última quinzena de agosto (cultivares mais precoces) até a primeira quinzena de janeiro (cultivares mais tardias). Esse padrão é esperado, uma vez que as atividades convectivas de MG e a atuação dos sistemas de Monção Sul-Americana (MSA) (Reboita et al. 2015) são responsáveis pelo clima típico de monção (inverno seco e verão chuvoso), influenciando nas variáveis $T m$ e $T M$ e $P$.

Houve variação na duração média da fase de desenvolvimento entre as cultivares, os subperíodos reprodutivos e os três anos agrícolas (Tabela 1). Porém, de maneira geral é possível observar a existência de uma relação inversa entre a duração de cada subperíodo e a temperatura média do ar. Isso significa que quanto menor (maior) a temperatura do ar, maior (menor) será a duração média de cada fase. Tal resposta já foi observada para culturas anuais como canola (Luz et al. 2012) e culturas perenes como eucalipto (Freitas et al. 2017) e cultivares de oliveira Arbequina (Martins et al. 2014), Grappolo e MGS Mariense (Souza e Martins, 2014), considerando o desenvolvimento vegetativo, representado pela fase de muda.

As variações nas condições meteorológicas foram importantes na estimativa da $T b$ para as diferentes 
cultivares de oliveira e subperíodos reprodutivos. Com exceção do $D P_{g d}$, os demais métodos foram coerentes em estimar a $T b$ entre as cultivares e subperíodos, com destaque para as metodologias tradicionais, principalmente $\mathrm{o}$ $C V_{g d}$, $C R$ e DR. Obviamente, mesmo com o zelo estatístico inerente a cada método de estimativa da $\mathrm{Tb}$, a utilização dos valores deve ser vista com cautela estando ciente dos erros que pode haver (Lago et al. 2009; Souza e Martins, 2014). Além disso, dependendo da espécie considerada, existem métodos que não conseguem estimar corretamente a $T b$ (Ferreira et al. 2019; Freitas et al. 2017).

Por brevidade, somente foi apresentado o valor final da $T b$ para cada cultivar e subperíodo reprodutivo, o qual foi considerado como a média da $T b$ obtida pelos nove métodos avaliados (Tabela 2). Alguns valores de $T b$ estimados foram maiores que $18{ }^{\circ} \mathrm{C}$, valor considerado não realístico biologicamente (Lago et al. 2009; Souza e Martins, 2014), sendo nesses casos substituído pela média aritmética da $T m$ do ar (Souza e Martins, 2014) durante o subperíodo correspondente.

Observou-se diferenças nos valores de $T b$ estimados entre as cultivares e subperíodo reprodutivo. Tal variação também foi constatada por Lago et al. (2009) para genótipos de arroz cultivado e biótipos de arroz vermelho, Souza et al. (2009) para a figueira, Pilau et al. (2011) para o crambe e Souza e Martins (2014) para cultivares de oliveira, o que é esperado, uma vez que existe diferença de valores de $T b$ entre espécies, genótipos de mesma espécie assim como o subperíodo de desenvolvimento em que a planta se encontra (Freitas et al. 2017; Lago et al. 2009; Streck et al. 2007).

Os valores estimados variaram para floração $\left(7,8^{\circ} \mathrm{C}\right.$ a $\left.16,0{ }^{\circ} \mathrm{C}\right)$, frutificação $\left(13,3{ }^{\circ} \mathrm{C}\right.$ a $\left.14,3{ }^{\circ} \mathrm{C}\right)$ e maturação $\left(7,1^{\circ} \mathrm{C}\right.$ a $17,2^{\circ} \mathrm{C}$ ) (Tabela 2). Os maiores valores de $T b$, assim como o maior intervalo de variação, ocorreram na maturação, que se estende desde janeiro até meados de março (Oliveira et al. 2012), possivelmente devido as maiores temperaturas médias do período (Tabela 1). Além disso, espera-se que os maiores valores de $T b$ ocorram na frutificação e maturação em função das necessidades fisiológicas da oliveira em ambos subperíodos (Garcia-Mozo et al. 2010; Wrege et al. 2015). Temperaturas elevadas - entre $25{ }^{\circ} \mathrm{C}$ e $35^{\circ} \mathrm{C}$ - são exigidas na frutificação (Wrege et al. 2015) e maturação (Coutinho et al. 2009; Garcia et al. 2018), que ocorre desde setembro a meados de novembro, para que haja o pleno desenvolvimento do fruto (GarciaMozo et al. 2010; Moriondo et al. 2015; Wrege et al. 2015).

A cultivar Arbequina apresentou $T b$ para a maturação menor que as demais cultivares $\left(7,1^{\circ} \mathrm{C}\right)$, sendo que uma provável justificativa seja a diferença entre os valores de $T b$ estimados pelas metodologias tradicionais e modificadas - de $2^{\circ} \mathrm{C}$ a $8,9^{\circ} \mathrm{C}$ (dados não mostrados).

Cabe ressaltar que os valores estimados para as dez cultivares foram próximos entre si considerando o mesmo subperíodo de desenvolvimento, exceto para Grappolo 561, Grappolo 575, Penafiel, Ropades 392 e Santa Catalina. Outros autores encontraram valores de $T b$ semelhantes aos deste estudo considerando cultivares, fases e subperíodos de desenvolvimento distintos. Por exemplo, Souza e Martins (2014) estimaram a Tb para o desenvolvimento vegetativo das cultivares Grappolo $\left(9,6^{\circ} \mathrm{C}\right)$ e Maria da Fé $\left(6,9{ }^{\circ} \mathrm{C}\right)$; Martins et al. (2012) para Arbequina $\left(10,5^{\circ} \mathrm{C}\right)$ e MGS ASC315 $\left(11^{\circ} \mathrm{C}\right)$ utilizando uma metodologia distinta das utilizadas neste estudo; Alcalá e Barranco (1992) encontraram a $\mathrm{Tb}$ entre 10 à $13{ }^{\circ} \mathrm{C}$ para a floração e Pérez-López et al. (2008) para a frutificação $\left(15^{\circ} \mathrm{C}\right)$ de diferentes cultivares de oliveira. Outros autores também reportaram valores de $T b$ semelhantes em outras espécies perenes, como a videira $\left(13,8^{\circ} \mathrm{C}\right.$ - florescimento à maturação), cultivares de café-arábica 'Rubi MG-1192' e 'Acaiá Cerrado MG-1474' (12,9 $\left.{ }^{\circ} \mathrm{C}\right)$ (Lima e Silva, 2008), e de maturação dos frutos de cultivares de manga 'Alfa' $\left(10^{\circ} \mathrm{C}\right)$ e 'Roxa' $\left(10,6^{\circ} \mathrm{C}\right)$ (Barros et al. 2010; Callejas et al. 2014).

O M2 apresentou o menor valor de CV, razão pela qual tal método foi escolhido como o mais indicado para descrever a necessidade térmica das dez cultivares de oliveira e subperíodos de desenvolvimento reprodutivo (Tabela 3) (Rosa et al. 2009; Streck et al. 2007). O M2 considera a comparação da $T b$ com a $T m$ do ar, no entanto, existem outros métodos de $S T a$ os quais consideram além da $T b$ as temperaturas ótimas e máximas das espécies (Rosa et al. 2009), os quais podem apresentar melhoria no cômputo da necessidade térmica e serem mais realísticos (Streck et al. 2009). Entretanto, devido a natureza dos dados fenológicos disponíveis nesse estudo, só foi possível a utilização do M1 e M2, os quais comparam a Tm e Tmed com a $T b$.

Os resultados da ANOVA para a variável STa demonstraram a existência de interação significativa $(p<0,05)$, entre as cultivares e subperíodos de desenvolvimento reprodutivo (Tabela 4). Independentemente da cultivar, o subperíodo que necessita maior acúmulo energético é a frutificação, seguida da floração e maturação. Além disso, as cultivares de oliveira possuem exigências térmicas distintas e variam de acordo com o subperíodo de desenvolvimento, corroborando com Gálan et al. (2001), Pérez-López et al. (2008) e Prela-Pantano et al. (2010).

Na floração as cultivares que necessitaram acumular menor quantidade de energia são: Santa Catalina, Ropades 392, Penafiel, Grappolo 575 e 561, não apresentando diferenças pelo teste de Scott-Knott (Tabela 4). Exceto para a Grappolo $541\left(\mathrm{~Tb}=8,9^{\circ} \mathrm{C}\right)$, o menor acúmulo pode ser explicado pelo maior valor de $T b$ para a floração (Tabela 2). Ao comparar a duração em dias do calendário civil com os valores de $S T a$ para a floração, observa-se que nem todas as cultivares que acumulam menor (maior) quantidade de energia apresentam menor (maior) duração. 
Tabela 2 - Valores médios de temperatura basal inferior $(T b)$ estimados pelas nove metodologias (tradicionais e modificações) para as dez cultivares de oliveira em cada subperíodo de desenvolvimento reprodutivo. Maria da Fé, MG (2008 a 2011).

\begin{tabular}{|c|c|c|}
\hline Genótipo & Subperíodo & Temperatura basal inferior $\left({ }^{\circ} \mathrm{C}\right)$ \\
\hline \multirow[t]{3}{*}{ Alto D’Ouro } & Floração & 7,8 \\
\hline & Frutificação & 14,2 \\
\hline & Maturação & 16,4 \\
\hline \multirow[t]{3}{*}{ Arbequina } & Floração & 8,6 \\
\hline & Frutificação & 13,3 \\
\hline & Maturação & 7,1 \\
\hline \multirow[t]{3}{*}{ Galega } & Floração & 10,7 \\
\hline & Frutificação & 14,3 \\
\hline & Maturação & 14,7 \\
\hline \multirow[t]{3}{*}{ Grappolo 541} & Floração & 8,9 \\
\hline & Frutificação & 14,1 \\
\hline & Maturação & 12,7 \\
\hline \multirow[t]{3}{*}{ Grappolo 561} & Floração & 15,1 \\
\hline & Frutificação & 14,1 \\
\hline & Maturação & 15,7 \\
\hline \multirow[t]{3}{*}{ Grappolo 575} & Floração & 14 \\
\hline & Frutificação & 13,6 \\
\hline & Maturação & 16,3 \\
\hline \multirow[t]{3}{*}{ Negroa } & Floração & 9,5 \\
\hline & Frutificação & 14,1 \\
\hline & Maturação & 16,1 \\
\hline \multirow[t]{3}{*}{ Penafiel } & Floração & 13,7 \\
\hline & Frutificação & 13,9 \\
\hline & Maturação & $17,2^{*}$ \\
\hline \multirow[t]{3}{*}{ Ropades 392} & Floração & 14,2 \\
\hline & Frutificação & 14 \\
\hline & Maturação & 16,7 \\
\hline \multirow[t]{3}{*}{ Santa Catalina } & Floração & 16 \\
\hline & Frutificação & 14,1 \\
\hline & Maturação & $16,1^{*}$ \\
\hline
\end{tabular}

*Valores substituídos pela média aritmética da temperatura mínima do ar referente a duração do subperíodo considerada, conforme recomendações de Souza e Martins (2014)

Como exemplo a cultivar Galega, que apresenta duração média de 52 dias e, no entanto, necessita acumular $394,58{ }^{\circ} \mathrm{C}$.dia, por outro lado, a cultivar Santa Catalina apresenta duração média maior (60 dias) e necessita acumular menor quantidade de energia $\left(264,52{ }^{\circ} \mathrm{C}\right.$. dia). Isso demonstra que a $S T a$ é mais coerente que o dia do calendário civil para avaliar a duração dos subperíodos de desenvolvimento reprodutivo de diferentes cultivares de oliveira, mesmo resultado observado para arroz (Carli et al. 2016; Streck et al. 2007), caju (Matos et al. 2014) e outras cultivares de oliveira (Pérez-López et al. 2008).

Valores de $S T a$ encontrados para a floração $(264,5 \leq$ Sta $\leq 629,6^{\circ} \mathrm{C}$.dia) são semelhantes aos reportados por Gálan et al. (2001) $\left(\approx 500{ }^{\circ} \mathrm{C}\right.$.dia); Melo-Abreu et al. (2004) $\left(460{ }^{\circ} \mathrm{C}\right.$.dia a $559^{\circ} \mathrm{C}$.dia) e inferior ao observado Pérez-López et al. (2008) $\left(\approx 800{ }^{\circ} \mathrm{C}\right.$.dia $)$ em diferentes cultivares de oliveira. Estudos recentes apontam que o fotoperíodo pode ter influência, embora menor que a temperatura e a $S T a$, na duração do florescimento da oliveira (Melo-Abreu et al. 2004; Orlandi et al. 2013). No entanto, ainda não há um consenso sobre a confirmação do efeito do fotoperíodo no desenvolvimento reprodutivo da oliveira (García-Mozo et al. 2009), a qual somente pode ser realizada mediante avaliação experimental, a qual é extremamente trabalhosa, onerosa, e em muitos casos, inviável na prática (Orlandi et al. 2013).

Com relação a frutificação, observa-se que todas as cultivares necessitam acumular quantidade similar de energia, variando entre $911,64{ }^{\circ} \mathrm{C}$.dia a $1128,79{ }^{\circ} \mathrm{C}$.dia para Grappolo 541 e Grappolo 575, respectivamente (Tabela 4). Valores de STa semelhantes podem ter ocorrido devido as semelhanças entre os valores de $T b$ para a frutificação (entre $13,3{ }^{\circ} \mathrm{C}$ e $14,3{ }^{\circ} \mathrm{C}$ ). No entanto, diferentemente da floração, na frutificação as cultivares que acumulam menor (maior) quantidade de energia são as que apresentam menor (maior) duração média, como exemplo a Alto D'ouro $\left(915,58{ }^{\circ} \mathrm{C} . \mathrm{dia} / 140\right.$ dias) e Grappolo 575 (1128,79 ${ }^{\circ} \mathrm{C}$. dia/173 dias). Vale ressaltar que considerando condições de temperaturas do ar amenas, o esperado é que quanto maior a necessidade térmica, maior é a duração (em dias do calendário civil) de uma determinada fase de desenvolvimento, já que será necessário um maior período de tempo para acumular a energia necessária (Aguilera et al. 2015; Rosa et al. 2009; Streck et al. 2007). Além disso, espera-se que o maior acúmulo energético ocorra na frutificação, pois grande parte da energia produzida na fotossíntese é alocada para a formação do fruto (Aguilera et al. 2015). Estudos que reportam sobre a $S T a$ durante a frutificação da oliveira são escassos. Dentre os raros trabalhos, Pérez-López et al. (2008) estimaram indiretamente a $S T a$, sem a utilização de dados fenológicos observados, e encontraram valores distintos aos deste estudo $\left( \pm 475^{\circ} \mathrm{C}\right.$. dia $)$.

Com relação à maturação, os valores de $S T a$ foram distintos entre as cultivares, variando entre $77,42{ }^{\circ} \mathrm{C}$.dia (Penafiel) e $482^{\circ} \mathrm{C}$.dia (Arbequina). Assim como observado na frutificação, as cultivares que apresentaram menor (maior) acúmulo energético tiveram, em média, menor (maior) duração em dias do calendário civil, conforme é esperado (Streck et al. 2007). Vale ressaltar, que a maturação é o subperíodo que, ao mesmo tempo, possui menor acúmulo térmico, porém, com as maiores $T b$ 's, exceto para a Arbequina $\left(7,1^{\circ} \mathrm{C}\right)$. 
Tabela 3 - Necessidade térmica, desvio padrão, coeficiente de variação para cada método (M1 e M2) e duração em dias do calendário civil para os três subperíodos de desenvolvimento reprodutivo das dez cultivares de oliveira. Maria da Fé, MG, 2008 à 2011.

\begin{tabular}{|c|c|c|c|c|c|c|c|c|c|}
\hline \multirow[t]{2}{*}{ Cultivar } & \multicolumn{3}{|c|}{ Floração } & \multicolumn{3}{|c|}{ Frutificação } & \multicolumn{3}{|c|}{ Maturação } \\
\hline & M1 & M2 & Dias & M1 & M2 & Dias & M1 & M2 & Dias \\
\hline \multicolumn{10}{|c|}{ 2008-2009 } \\
\hline$\overline{\text { Alto D’Ouro }}$ & 525,3 & 552,4 & 60 & 758,1 & 870,8 & 148 & 81,7 & 94,6 & 17 \\
\hline Arbequina & 435,7 & 473,7 & 56 & 864,9 & 982,5 & 153 & 457,8 & 457,8 & 26 \\
\hline Galega & 324,8 & 365,7 & 49 & 819,8 & 895,4 & 146 & 125,6 & 128,3 & 17 \\
\hline Grappolo 541 & 409,2 & 451,6 & 54 & 772,2 & 881,8 & 148 & 206,7 & 207,1 & 23 \\
\hline Grappolo 561 & 147,0 & 351,4 & 80 & 799,9 & 893,2 & 146 & 64 & 72,5 & 11 \\
\hline Grappolo 575 & 97,4 & 245,5 & 53 & 965,5 & 1076,8 & 168 & 103,3 & 116,6 & 18 \\
\hline Negroa & 421,6 & 473,9 & 60 & 772,2 & 881,8 & 148 & 112,9 & 124,5 & 21 \\
\hline Penafiel & 132,9 & 266,9 & 50 & 743,5 & 853,6 & 142 & 97,0 & 123,1 & 24 \\
\hline Ropades 392 & 141,2 & 288,3 & 56 & 812,8 & 926,8 & 153 & 78,0 & 104,4 & 21 \\
\hline Santa Catalina & 141,6 & 312,8 & 70 & 828,7 & 928,3 & 153 & 135,2 & 147,3 & 23 \\
\hline \multicolumn{10}{|c|}{ 2009-2010 } \\
\hline Alto D'Ouro & 635,3 & 654,0 & 66 & 935,8 & 978,6 & 138 & 88,7 & 100,3 & $\overline{15}$ \\
\hline Arbequina & 477,3 & 502,1 & 56 & 1060,9 & 1119,5 & 148 & 574,9 & 574,9 & 31 \\
\hline Galega & 350,2 & 399,3 & 57 & 990,9 & 1059,6 & 155 & 179,0 & 183,2 & 25 \\
\hline Grappolo 541 & 667,9 & 705,6 & 82 & 928,8 & 900,3 & 143 & 172,1 & 172,1 & 18 \\
\hline Grappolo 561 & 131,2 & 340,2 & 85 & 1016,1 & 1108,3 & 162 & 133,9 & 143,3 & 20 \\
\hline Grappolo 575 & 199,4 & 388,9 & 85 & 1174,8 & 1185,1 & 179 & 53,6 & 59,8 & 13 \\
\hline Negroa & 479,7 & 513 & 61 & 983,7 & 1023,0 & 140 & 91,8 & 98,0 & 15 \\
\hline Penafiel & 239,9 & 371,5 & 72 & 1136,0 & 1210,4 & 168 & 64 & 74,6 & 23 \\
\hline Ropades 392 & 197,1 & 328,5 & 66 & 1059,0 & 1116,8 & 157 & 75,0 & 92,7 & 16 \\
\hline Santa Catalina & 129,9 & 293,4 & 69 & 1107,4 & 1164,5 & 163 & 66,2 & 77,4 & 16 \\
\hline \multicolumn{10}{|c|}{$2010-2011$} \\
\hline$\overline{\text { Alto D’Ouro }}$ & 511,9 & 529,7 & 51 & 821,2 & 897,3 & 133 & 58,3 & 71,0 & $\overline{11}$ \\
\hline Arbequina & 512,0 & 577,7 & 65 & 977,9 & 1105,5 & 155 & 413,3 & 413,3 & 22 \\
\hline Galega & 361,1 & 418,7 & 51 & 866,5 & 959,3 & 146 & 143,8 & 148,0 & 21 \\
\hline Grappolo 541 & 649,2 & 731,3 & 84 & 898,4 & 952,7 & 152 & 162,1 & 162,1 & 19 \\
\hline Grappolo 561 & 99,8 & 208,6 & 45 & 834,6 & 908,0 & 133 & 79,1 & 88,9 & 13 \\
\hline Grappolo 575 & 113,3 & 186,1 & 35 & 1039,1 & 1124,4 & 173 & 80,9 & 91,6 & 15 \\
\hline Negroa & 423,5 & 462,7 & 51 & 853,2 & 933,5 & 140 & 94,2 & 106,8 & 16 \\
\hline Penafiel & 163,0 & 226,6 & 36 & 1003,4 & 1079,5 & 156 & 31,0 & 34,4 & 6 \\
\hline Ropades 392 & 161,09 & 234,15 & 39 & 892,13 & 970,03 & 143 & 63,63 & 89,63 & 16 \\
\hline Santa Catalina & 89,49 & 187,3 & 40 & 973,96 & 1081,56 & 160 & 68,25 & 76,1 & 12 \\
\hline Desvio Padrão & 187,4 & 149,9 & 14,4 & 118,6 & 107,1 & 11,2 & 125,4 & 121,6 & 5,3 \\
\hline CV $(\%)$ & 60 & 37,3 & 24,2 & 12,8 & 10,7 & 7,4 & 90,5 & 82,3 & 29,2 \\
\hline
\end{tabular}

A quantificação do tempo térmico necessário para atingir cada fase de desenvolvimento são ferramentas importantes no manejo das culturas (Ferreira et al. 2019; Carli et al. 2016; Rosa et al. 2009), principalmente durante os subperíodos de desenvolvimento reprodutivo, os quais necessitam de maior planejamento para a aplicação dos tratos culturais. No caso da oliveira, essa quantificação, inédita para as condições climáticas brasileiras, são úteis para definir o melhor momento para a aplicação de fungicidas e sprays profiláticos (Pérez-López et al. 2008), evitando assim, a ocorrência e a proliferação de doenças no fruto da oliveira, como Spilocaea oleaginosa,
Pseudocercospora cladosporioides e Gloeosporium olivae (Garcia et al. 2018; Wrege et al. 2015). Além disso, vários estudos têm demonstrado que a fenologia e o momento de ocorrência de cada fase do desenvolvimento reprodutivo é um importante indicador dos impactos das mudanças climáticas na oliveira (García-Mozo et al. 2010; Moriondo et al. 2015), principalmente pela dependência da temperatura do ar (Aguilera et al. 2015; Martins et al. 2014; PérezLópez et al. 2008).

De maneira geral, observam-se que as cultivares mais precoces, as quais acumulam menor quantidade de energia e ao mesmo tempo tem menor duração (dias do 
Tabela 4 - Comparação de médias para a necessidade térmica (STa, ${ }^{\circ} \mathrm{C}$.dia) calculados pelo Método 2 e duração média, em dias do calendário civil, para as dez cultivares de oliveira nos três subperíodos de desenvolvimento reprodutivo. Maria da Fé, MG, 2008 a 2011.

\begin{tabular}{|c|c|c|c|c|c|c|}
\hline Cultivar & Floração & Duração média (dias)* & Frutificação & Duração média (dias)* & Maturação & Duração média (dias)* \\
\hline Alto D’Ouro & $578,72 \mathrm{bB}$ & 59 & $915,58 \mathrm{cA}$ & 140 & $88,64 \mathrm{aA}$ & 14 \\
\hline Arbequina & $517,87 \mathrm{aB}$ & 59 & $1069,22 \mathrm{bA}$ & 152 & $482,03 \mathrm{aC}$ & 26 \\
\hline Galega & $394,58 \mathrm{bB}$ & 52 & $971,47 \mathrm{cA}$ & 149 & $153,19 \mathrm{aB}$ & 21 \\
\hline Grappolo 541 & $629,55 \mathrm{bB}$ & 73 & $911,64 \mathrm{cA}$ & 148 & $180,43 \mathrm{aB}$ & 20 \\
\hline Grappolo 561 & $300,1 \mathrm{bA}$ & 60 & $969,9 \mathrm{cA}$ & 147 & $101,58 \mathrm{aA}$ & 15 \\
\hline Grappolo 575 & $273,52 \mathrm{bA}$ & 58 & $1128,79 \mathrm{cA}$ & 173 & $89,37 \mathrm{aA}$ & 15 \\
\hline Negroa & $483,2 \mathrm{bB}$ & 57 & $946,15 \mathrm{cA}$ & 143 & $109,83 \mathrm{aA}$ & 17 \\
\hline Penafiel & $288,35 \mathrm{bA}$ & 53 & $1047,86 \mathrm{cA}$ & 155 & $77,42 \mathrm{aA}$ & 14 \\
\hline Ropades 392 & $283,68 \mathrm{bA}$ & 53 & $1004,58 \mathrm{cA}$ & 151 & $95,59 \mathrm{aA}$ & 18 \\
\hline Santa Catalina & $264,52 \mathrm{bA}$ & 60 & $1058,14 \mathrm{cA}$ & 159 & $100,3 \mathrm{aA}$ & 17 \\
\hline
\end{tabular}

Médias seguidas pela mesma letra minúscula na linha e maiúscula na coluna não diferem entre si pelo teste Scott Knott a 5\% de probabilidade. As letras foram dadas em ordem decrescente de soma térmica acumulada para cada subperíodo correspondente. *Valores extraídos de Oliveira et al. (2012).

calendário civil), são: Santa Catalina, Ropades 392, Penafiel e Grappolo 561 (Fig. 1). As cultivares Arbequina e Grappolo 541 são as que precisam acumular maior quantidade de energia. No caso da Arbequina, tal resultado provavelmente se deve aos menores valores de $T b$ nos três subperíodos, em especial na maturação $\left(7,1^{\circ} \mathrm{C}\right)$, o que the permite acumular energia mesmo em dias com temperaturas amenas.

No entanto, considerando os dias do calendário civil não é possível observar diferenças entre as cultivares. Vale lembrar que, de acordo com a metodologia da $S T a$, quão maior for a diferença entre a Tmed e a $T b$ da cultivar, maior será o acúmulo térmico diário. Assim, $T b$ 's menores irão promover maior acúmulo em comparação com $T b$ 's elevadas, caso estejam sob as mesmas condições meteorológicas. Existem outros métodos de $S T d_{i}$, os quais penalizam de diferentes maneiras a Tmed com as três temperaturas cardinais (Tb, Tot e $T B$ ) (Freitas e Martins, 2019; Lago et al. 2009; Rosa et al. 2009). No entanto, devido aos dados disponíveis nesse estudo, só foi possível a utilização dos M1 e M2, os quais consideram apenas a Tb (Lago et al. 2009; Souza e Martins, 2014).

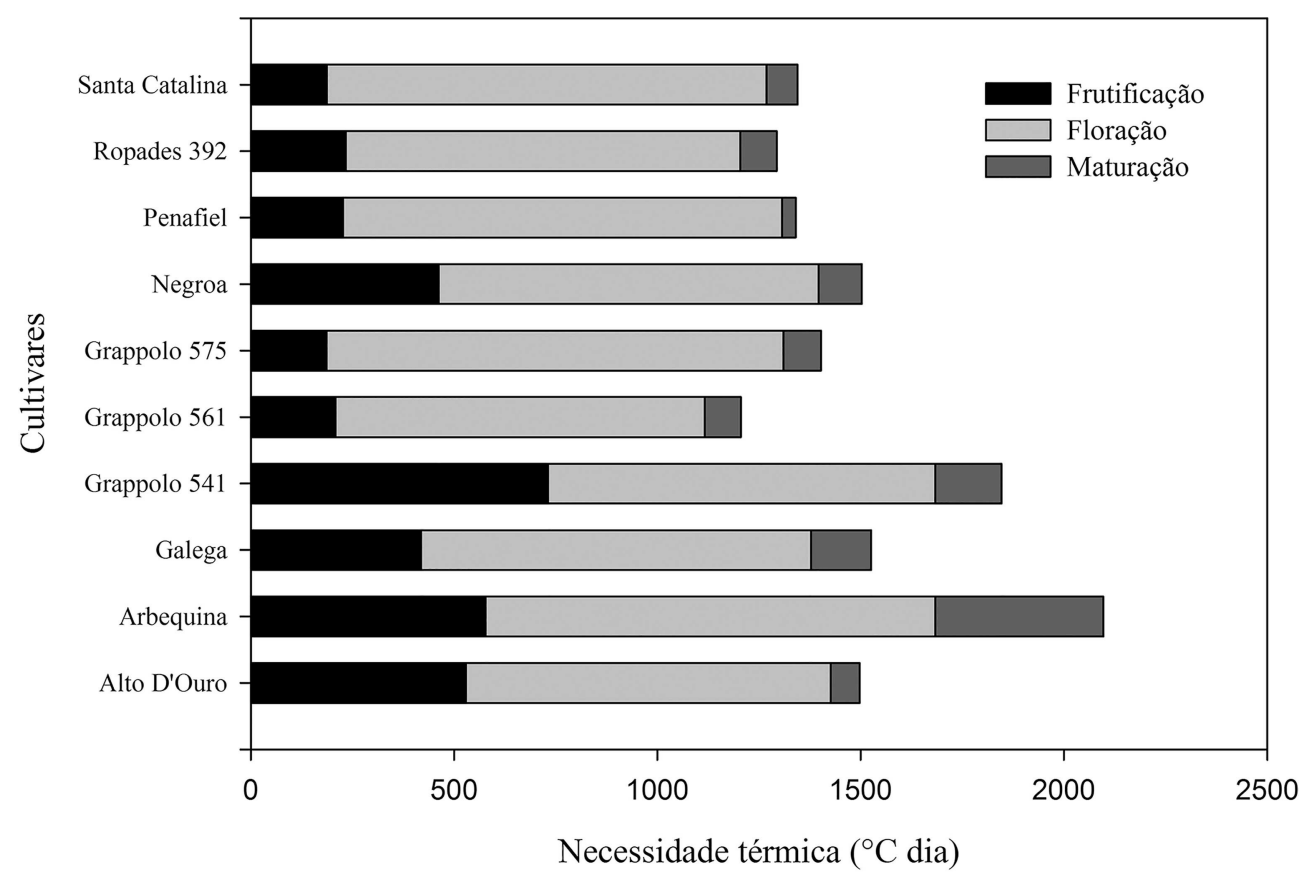

Figura 1. - Necessidade térmica acumulada média nos subperíodos de desenvolvimento reprodutivo obtido pelo Método 2. Maria da Fé, MG, 2008 a 2011. 
Analisando todas as cultivares, em média, a floração demanda $26 \%$ da energia total necessária para o desenvolvimento reprodutivo, seguido da frutificação (66\%) e maturação ( $8 \%$ ). Informações básicas sobre o desenvolvimento reprodutivo, como a estimativa da $T b$ e a necessidade térmica, em cultivares de oliveira são fundamentais no conhecimento das cultivares mais adaptadas às condições climáticas de cultivo e as necessidades da cultura em condições meteorológicas distintas do seu centro de origem (Martins et al. 2012; Souza e Martins, 2014). Além disso, dão subsídio na determinação do momento exato ocorrência dos subperíodos reprodutivos, a qual possibilita o controle de doenças e pestes na oliveira, como exemplo a aplicação de defensivos contra a mosca da azeitona (Bactrocera oleae Gmel.), traça da oliveira (Prays oleae Bern.) sprays profiláticos - aumentam a produtividade (Pérez-López et al. 2008).

\section{Conclusões}

Houve variação dos valores de $T b$ 's e necessidade térmica entre as cultivares e subperíodos de desenvolvimento reprodutivo. As $T b$ 's variaram para a floração de $7,8{ }^{\circ} \mathrm{C}$ a $16,1{ }^{\circ} \mathrm{C}$, para a frutificação de $13,3{ }^{\circ} \mathrm{C}$ a $14,3{ }^{\circ} \mathrm{C}$, e maturação de $7,1^{\circ} \mathrm{C}$ a $17,2^{\circ} \mathrm{C}$. Já a frutificação é o subperíodo que demanda maior necessidade térmica, com valores variando entre $911,64^{\circ} \mathrm{C}$.dia a $1128,79^{\circ} \mathrm{C}$.dia.

As cultivares mais precoces, as quais acumulam menor quantidade de energia e possuem menor duração em dias, são: Santa Catalina, Ropades 392, Penafiel e Grappolo 561, e as cultivares Arbequina e Grappolo 541 são as mais tardias.

\section{Agradecimentos}

À Fundação de Amparo à Pesquisa de Minas Gerais (FAPEMIG) pelo auxílio financeiro referente aos projetos APQ 01392-13 e APQ 01258-17. À Coordenação de Aperfeiçoamento de Pessoal de Nível Superior (CAPES, processo número 1489491) pela bolsa concedida ao segundo autor.

\section{Referências}

AGUILERA, F.; ORLANDI, F.; RUIZ-VALENZUELA, L.; MSALLEM, M.; FORNACIARI, M. Analysis and interpretation of long temporal trends in cumulative temperatures and olive reproductive features using a seasonal trend decomposition feature. Agricultural and Forest Meteorology, v. 203, p. 208-216, 2015.

ALCALÁ, A.R.; BARRANCO, D.; Prediction off lowering time in olive for the Cordoba olive collection. Horticultural Science, v. 27, n. 11, p. 1205-1207, 1992.

BARROS, M.P.; ZANETTI, V.B.; FRAGA, C.I.M.; NINCE, P.C. C.; CAMPELO JÚNIOR, J.H.; LOBO, F.A. Unidades fototérmicas e temperatura-base inferior de frutos de mangueira alfa, na Baixada Cuiabana. Revista Brasileira de Fruticultura, v. 32, n. 2, p. 479-485, 2010.

CALLEJAS, I.J.A.; NEVES, G.A.R.; TAVARES, A.S.; MOURA, I.B.; LIMA, E.A. Determinação das temperaturas cardinais da manga cultivar Roxa através de simulação computacional utilizando um modelo não linear. Ambiência v. 10, n. 1, p. 97-110, 2014.

CARLI, C.; STEINMETZ, S.; STRECK, N.A.; MARCHESAN, E.; SILVA; M.R. Número de dias e de graus-dia entre a iniciação e a diferenciação da panícula em cultivares de arroz irrigado. Ciência Rural, v. 46, n. 3, p. 428-433, 2016.

COUTINHO, E.F.; RIBEIRO, F.C.; CAPPELLARO, T.H. Cultivo de Oliveira (Olea europaea L.). Pelotas: Embrapa Clima Temperado, 2009. 125p. (Embrapa Clima Temperado. Sistemas de produção, 16). Disponível em: https:// www.infoteca.cnptia.embrapa.br/infoteca/bitstream/doc/ 783494/1/sistema16.pdf. Acesso em 27 de fevereiro de 2018.

FAGUNDES, J.D.; STRECK, N.A.; STORCK, L.; REINIGER, L.R.S.; Temperatura-base e soma térmica de subperíodos do desenvolvimento de Aspilia montevidensis. Bragantia, v. 39, n. 2, p. 499-509, 2010.

FERREIRA, D.F. Sisvar: a computer statistical analysis system. Ciência e Agrotecnologia, v. 35, n. 6, p. 1039-1042, 2011.

FERREIRA, M.C.; MARTINS, F.B.; FLORÊNCIO, G.W.L.; SILVA, J.P.G.C.; PASIN, L.A.A.P. Temperaturas cardinais e necessidade térmica no desenvolvimento inicial de duas espécies nativas brasileiras. Pesquisa Agropecuária Brasileira, 2019. no prelo.

FREITAS, C.H.de; MARTINS, F.B.; ABREU, M.C. Temperaturas cardinais para o desenvolvimento foliar de mudas de Corymbia citriodora e Eucalyptus urophylla. Pesquisa Agropecuária Brasileira, v. 52, n. 5, p. 283-292, 2017.

FREITAS, C.H.de; MARTINS, F.B. Métodos de soma térmica e influência do fotoperíodo no desenvolvimento foliar de duas espécies florestais. Floresta e Ambiente, 2019, no prelo.

GALÁN, C., GARCÍA-MOZO, H., CARIÑANOS, P., ALCÁZAR, P., DOMÍNGUEZ-VILCHES, E. The role of temperature in the onset of the Olea europaea L. pollen season in southwestern Spain. International Journal of Biometeorology, v. 45, n. 1, p. 8-12, 2001.

GARCÍA-MOZO, H.; MESTRE, A. GALÁN, C. Phenological trends in southern Spain: A response to climate change. Agricultural and Forest Meteorology, v. 150, p. 575-580, 2010.

GARCÍA-MOZO, H.; ORLANDI, F. GALÁN, C.; FORNACIARI, M.; ROMANO, B.; RUIZ, L.; DIAZ de La GUARDIA, C.; TRIGO, M.M.; CHUINE, I. Olive flowering phenology variation between different cultivars in Spain and Italy: modeling analysis. Theoretical and Applied Climatology, v. 95, p. 385-395, 2009.

GARCIA, S.R.; SANTOS, D.F.dos; MARTINS, F.B.; TORRES, R.R. Aspectos climatológicos associados ao cultivo da oliveira (Olea europaea L.) em Minas Gerais. Revista Brasileira de Climatologia, v. 22, p. 188-209, 2018.

LAGO, I.; STRECK, N.A.; CARVALHO, M.P.; FAGUNDES, L. K.; PAULA, G.M.de.; LOPES, S.J. Estimativa da temperatura base do subperíodo emergência-diferenciação da 
panícula em arroz cultivado e arroz vermelho. Revista Ceres, v. 56, n. 3, p. 288-295, 2009.

LIMA, E.P.; SILVA, E.L.da. Temperatura base, coeficientes de cultura e graus-dia para cafeeiro arábica em fase de implantação. Revista Brasileira de Engenharia Agrícola e Ambiental, v. 12, n. 3, p. 266-273, 2008.

LUZ, G.L.da; MEDEIROS, S.L.P.; TOMM, G.O.; BIALOZOR, A.; AMARAL, A.D.do.; PIVOTO, D. Temperatura base inferior e ciclo de híbridos de canola. Ciência Rural, v. 42, n. 9, p. 1549-1555, 2012.

MARTINS, F.B.; PEREIRA, R.A. A.; PINHEIRO, M.V.M.; ABREU, M.C. Desenvolvimento foliar em duas cultivares de oliveira estimado por duas categorias de modelos. Revista Brasileira de Meteorologia, v. 29, n. 4, p. 505514, 2014.

MARTINS, F.B.; REIS, D.F.; PINHEIRO, M.M.V. Temperatura base e Filocrono em duas cultivares de oliveira. Ciência Rural, v. 42, n. 11, p. 1975-1981, 2012.

MATOS, V.A.T.M; PIVETTA, F.; SOBRINHO, S.P.; TISSIANI, A.S.O; PEREIRA, A.P.M.S; RAMOS, F.T.; JÚNIOR, J.H. C.; Temperaturas basais e exigência térmica para a maturação de caju. Bioscience Journal, v. 30, n. 4, p. 969-977, 2014.

MELO-ABREU, J.P., BARRANCO D., CORDEIRO A.M., TOUS J., ROGADO B.M., VILLALOBOS F.J., Modelling olive flowering date using chilling for dormancy release and thermal time, Agricultural and Forest Meteorology, v. 125, p. 117-127, 2004.

MORIONDO, M.; FERRISE, R.; TROMBI, G.; BRILLI, L.; DIBARI, C.; BINDI. M. Modelling olive trees and grapevines in a changing climate. Environmental Modelling \& Software, v. 72, p. 387-401, 2015.

OLIVEIRA, M.C.; RAMOS J.D.; PIO R.; CARDOSO M.C. Características fenológicas e físicas e perfil de ácidos graxos em oliveiras no sul de Minas Gerais. Pesquisa Agropecuária Brasileira, v. 47, n. 1, p. 30-35, 2012.

ORLANDI, F.; GARCÍA-MOZO, H.; DHIAB, A.B.; GALÁN, C.; MSALlEM, M.; ROMANO, B.; ABICHOU, M.; DOMINGUES-VILCHES, E.; FORNACIARI, M. Climatic indices in the interpretation of the phonological phases of the olive in Mediterranean areas during its biological cycle. Climatic Change, v. 116, p. 263-284, 2013.

PÉREZ-LÓPEZ, D., RIBAS, F., MORIANA, A., RAPOPORT, H. F.; DE JUAN, A. Influence of temperature on the growth and development of olive (Olea europaea L.) trees. The Journal of Horticultural Science and Biotechnology, v. 83, p. 171-176, 2008.

PILAU, G.V.; BATTISTI, R.; SOMAVILLA, L.; SCHWERZ, L.; Temperatura basal, duração do ciclo e constante térmica para a cultura do crambe. Bragantia, v. 70, n. 4, p. 958964, 2011.

PRELA-PANTANO A.; TERAMOTO, J.R.S.; BERTONCINI, E.I. Análises preliminares das condições climáticas do estado de São Paulo para o cultivo de oliveiras. 2010. Disponível em: http://www.infobibos.com/Artigos/2010_3/ ClimaOliva/index.htm. Acesso em 27 de fevereiro de 2018.

REBOITA, M.S.; RODRIGUES, M.; SILVA, L.F.; ALVES, M.A. Aspectos climáticos do Estado de Minas Gerais.
Revista Brasileira de Climatologia, v. 17, p. 206-226, 2015.

ROSA, H.T. WALTER, L.C.; STRECK, N.A.; ALBERTO, C.M. Métodos de soma térmica e datas de semeadura na determinação de Filocrono de cultivares de trigo. Pesquisa Agropecuária Brasileira, v. 44, n. 11, p. 1374-1382, 2009.

SANZ-CORTÉS, F.; MARTINEZ-CALVO, J.; BADENES, M. L.; BLEIHOLDER, H.; HACK, H.; LLACER, G.; MEIER, U. Phenological growth stages of olive trees (Olea europaea L.). Annals of Applied Biology, v. 140, n. 2, p. 151$157,2002$.

SILVA, E.D. ; REBOITA, M.S. Estudo da Precipitação no Estado de Minas Gerais-MG. Revista Brasileira de Climatologia, v. 13, p. 120-136, 2014.

SILVA, L.F.O.; OLIVEIRA, A.F.; PIO, R.; ZAMBON, C.R. Caracterização agronômica e carpométrica de cultivares de oliveira. Pesquisa Agropecuária Tropical, v. 42, n. 3, p. 350356, 2012.

SOUZA, A.P.S; LEONEL, S.; ESCOBEDO, J.F. Temperaturas basais e soma térmica para a figueira podada em diferentes épocas. Revista Brasileira de Fruticultura, v. 31, n. 2, p. 314-322, 2009.

SOUZA, P.M.B.; MARTINS, F.B.; Estimativa da temperatura basal inferior para as cultivares de oliveira Grappolo e Maria da Fé. Revista Brasileira de Meteorologia, v. 29, n. 2, p. 307-313, 2014.

STRECK, N.A. PAULA, F.L.M.de; BISOGNIN, D.A.; HELDWEIN, A.B.; DELLAI, J. Simulating the development of field-grown potato (Solanum tuberosum L.). Agricultural and Forest Meteorology, v. 141, p. 1-11, 2007.

STRECK, N.A.; GABRIEL, L.F.; SAMBORANHA, F.K.; LAGO, I.; SCHWANTES, A.P.; SCHONS, A. Comparing two versions of a non-linear model for simulating leaf number and developmental stages in maize based on air temperature. Ciência Rural, v. 39, n. 3, p. 642-648, 2009.

TRADEMAP. List of importers for the selected product. Product: 1509 Olive oil and its fractions obtained from the fruit of the olive tree solely by mechanical or other physical means under conditions that do not lead to deterioration of the oil, whether or not refined, but not chemically modified. 2018. Disponível em: https://www. trademap.org/Country_SelProduct_TS.aspx?nvpm=1|||| 150910||6|1|1|1|2|1|2|2|1. Acesso em 12 de março de 2018.

VIEIRA NETO, J.; OLIVEIRA, A.F.de; OLIVEIRA, N.C.de; DUARTE, H.S.S.; GONÇALVES, E.D. Aspectos técnicos da cultura da oliveira. Belo Horizonte: EPAMIG, 2008. 56p. (Boletim Técnico, 88).

WREGE, M.S; COUTINHO, F.E; PANTANO, A.P.; JORGE, R. O.; Distribuição Potencial de oliveiras no Brasil e no mundo. Revista Brasileira de Fruticultura, v. 37, n. 3, p. 656-666, 2015.

YANG S.; LOGAN, J.; COFFEY D.L. Mathematical formulae for calculating the base temperature for growing degree days. Agricultural and Forest Meteorology, v. 75, p. 6174, 1995.

This is an Open Access article distributed under the terms of the Creative Commons Attribution License, which permits unrestricted use, distribution, and reproduction in any medium, provided the original work is properly cited. 